JPET \# 111245

\title{
Transport, Metabolism, and In Vivo Population Pharmacokinetics of the Chloro Benztropine Analogs; a Class of Compounds Extensively Evaluated in Animal Models of Drug Abuse
}

\author{
Ahmed A. Othman, Shariq A. Syed, Amy H. Newman and Natalie D. Eddington
}

Pharmacokinetics-Biopharmaceutics Laboratory, Department of Pharmaceutical Sciences, School of Pharmacy, University of Maryland Baltimore (A.A.O., S.A.S., N.D.E.), Medicinal Chemistry Section, National Institute on Drug Abuse- Intramural Research Program, National Institute of Health (A.H.N.), Baltimore, MD, USA 
a) Running title: Transport, Metabolism and PK of the Chloro BZT Analogs.

b) Corresponding author: Dr. Natalie D. Eddington, Department of Pharmaceutical Sciences, School of Pharmacy, University of Maryland Baltimore, 20 Penn Street, HSF-2, Baltimore, MD, 21201. Tel: (410) 706- 6710; Fax: (410) 706-5017;

Email: neddingt@rx.umaryland.edu

c) Text pages: 25

Tables: 5

Figures: 5

References: 32

Words in abstract: 248

Words in introduction: 617

Words in discussion: 1500

Non standard abbreviations:

BZT, benztropine;DA, dopamine; DAT, dopamine transporter; BBB, blood brain barrier; HLM, human liver microsomes; RLM, rat liver microsomes; Papp, apparent permeability coefficient; A-B, apical to basolateral; B-A, basolateral to apical; P-gp, P-glycoprotein; PK, pharmacokinetics; AUC, area under the curve; Vss, steady state volume of distribution.

d) Recommended section assignment: Metabolism, Transport, and Pharmacogenomics 
JPET \# 111245

\section{ABSTRACT}

Recently extensive behavioral research has been conducted on the benztropine (BZT) analogs with the goal of developing successful therapeutics for cocaine abuse. The present study was conducted to characterize the contribution of dispositional factors in mediating the behavioral differences among the chloro BZT analogs and to identify cytochrome P450 enzymes involved in their metabolism. Bidirectional transport and efflux studies of four of the chloro BZT analogs were conducted. Screening with a panel of human and rat supersomes ${ }^{\mathrm{TM}}$ was performed for 4`,4“-diCl BZT. In addition, pharmacokinetic and brain distribution studies for 4`-Cl and 4`,4“-diCl BZT in SpragueDawley rats were conducted. The permeability of the chloro analogs ranged from 8.2632.23 and 1.37-21.65 $\times 10^{-6} \mathrm{~cm} / \mathrm{sec}$, while the efflux ratios ranged from 2.1-6.9 and 3.328.4 across MDCK-MDR1 and Caco-2 monolayers, respectively. The Pgp inhibitor verapamil reduced the efflux ratios and enhanced the absorptive transport of the chloro BZT analogs. 4`,4“-diCl BZT was a substrate of human CYP 2D6, 2C19, rat 2C11 and 3A1. The brain uptake for $4{ }^{`}-\mathrm{Cl}$ and $4{ }^{\prime}, 4^{\prime \prime}-\mathrm{diCl} \mathrm{BZT}$ was comparable and higher than previously reported for cocaine $\left(\mathrm{Ri}=4.6-4.7\right.$ vs 2.1 for cocaine). The rank order for $\mathrm{t}_{12}$ was 4,4“-diCl BZT >> 4`-Cl BZT > cocaine and for Vss was 4`-Cl BZT > 4`,4“-diCl BZT >> cocaine. In conclusion, the chloro analogs differ significantly in their clearance and duration of action which correlates to their behavioral profiles and abuse liability. Further, these results suggest that the distinctive behavioral profile of these analogs is not due to limited brain exposure. 
JPET \# 111245

\section{INTRODUCTION}

The psychostimulant effects of cocaine are believed to be mediated mainly through inhibition of dopamine uptake via the dopamine transporter (DAT) (Ritz et al., 1987; Volkow et al., 1997). Accordingly, the DAT has been regarded as one of the key targets for the development of cocaine agonist therapeutics (Carroll et al., 1999; Rothman et al., 2005). Benztropine (BZT) is a potent dopamine uptake inhibitor that is not subject to significant abuse in humans (Rothman, 1990). As such, BZT was used as the pharmacophore for the design of a series of BZT analogs with the purpose of enhancing the potency and selectivity towards DAT (Newman and Kulkarni, 2002; Zou et al., 2003; Kulkarni et al., 2004). The overall goal of these studies has been to better understand the role of DAT in cocaine addiction and to guide the design of ligands that may serve as medications for the treatment for cocaine abuse.

The BZT analogs have shown lower abuse potential in comparison to cocaine (Newman et al., 1994; Woolverton et al., 2001; Katz et al., 2004). However, they vary in terms of their relative efficacies in certain preclinical behavioral assays. In a cocaine discrimination study in rats, 4-Cl BZT (4`-chloro-3 $\alpha$-(diphenylmethoxy)tropane) had a maximal effect of $54.9 \%$ of cocaine appropriate responses while the maximal effect for 4,4“-diCl BZT (4`4“'-dichloro-3 $\alpha$-(diphenylmethoxy)tropane) was $17.6 \%$ (Katz et al., 1999). It was also reported that $44^{`} \mathrm{Cl}$ BZT and $3^{`}-\mathrm{Cl}$ BZT (3`-chloro-3 $\alpha-$ (diphenylmethoxy)tropane) were the most effective in stimulating locomotor activity of mice in a one hour observation and in pretreatment studies, followed by $3{ }^{\prime}, 4{ }^{\prime \prime}-\mathrm{diCl} \mathrm{BZT}$ (3`4“-dichloro-3 $\alpha$-(diphenylmethoxy)tropane) while 4`4“-diCl BZT only marginally increased locomotor activity (Katz et al., 2001). The same report indicated that the 
maximal stimulation of locomotor activity occurred first for $3 `-\mathrm{Cl}$ followed by $4{ }^{`}-\mathrm{Cl}$ then 3`,4“-diCl BZT. Self administration studies in rhesus monkeys showed that the rank order for reinforcing effectiveness of the chloro analogs was $3 `-\mathrm{Cl} \mathrm{BZT}=4{ }^{`}-\mathrm{Cl} \mathrm{BZT}>>$ 3`,4“-diCl BZT, in agreement with the locomotor data (Woolverton et al., 2001). The fact that the binding and potency profiles of the four chloro analogs at DAT are comparable (Table 1) suggests the involvement of other factors that may explain the discrepancy between binding at DAT and behavior including possible dispositional factors (Woolverton et al., 2000). These dispositional factors may include differences among the analogs in the rate and/or extent of brain exposure at equivalent doses, differences in their permeability across the blood brain barrier (BBB), different affinity for efflux transporters expressed at the BBB, or differences in the elimination half-life and consequently the duration of action.

The present studies were designed to evaluate the possible contribution of dispositional factors in mediating the reported differences in the behavioral pharmacology of the chloro analogs (as compared to one another and to cocaine) and to characterize the metabolic stability as well as the human and rat cytochrome P450 enzymes involved in their metabolism. No previous studies have been reported to characterize the enzymes involved in the metabolism of benztropine or the BZT analogs, even though the metabolites of benztropine were detected in rat urine and bile more than a decade ago (He et al., 1995). Consequently, the human and rat cytochrome P450 enzymes involved in the metabolism of 4',4"-diCl BZT were screened as an initial step to understand the elimination mechanisms of this class of compounds. In addition, the in vitro permeability and the possible $\mathrm{P}$-glycoprotein ( $\mathrm{P}-\mathrm{gp}$ ) interaction of the chloro 
analogs using two different cell lines was assessed. The goal was to examine whether these analogs differ significantly in permeability and efflux. Based on the results of this evaluation, we selected two analogs and compared their in vivo pharmacokinetic and brain uptake profiles in Sprague Dwaley rats. 
JPET \# 111245

\section{MATERIALS AND METHODS}

\section{MATERIALS}

Caco-2 cells were purchased from ATCC (Rockville, MD). MDCK- MDR1 cells were a gift from Dr. Peter Swaan (University of Maryland Baltimore, MD). Cell culture supplies (Dulbecco's modified Eagle medium (DMEM), phosphate buffered saline with $\mathrm{Ca}^{2+}$ and $\mathrm{Mg}^{2+}(\mathrm{PBS})$, L-glutamine (100X), non essential amino acids, fetal bovine serum, trypsin $0.25 \%$-EDTA $(1 \mathrm{mM})$, and penicillin $\mathrm{G}$ - streptomycin sulfate antibiotic mixture) were purchased from Gibco Laboratories (Lenexa, KS). $\left[{ }^{14} \mathrm{C}\right]$ Mannitol (46 mCi/mmol), $\left[{ }^{14} \mathrm{C}\right]$ paclitaxel $(52.3 \mathrm{mCi} / \mathrm{mmol}),\left[{ }^{3} \mathrm{H}\right]$ propranolol $(20 \mathrm{mCi} / \mathrm{mmol})$, sodium phosphate dibasic, verapamil $\mathrm{HCl}$ and oxprenolol were purchased from Sigma Chemical Co. (St. Louis, MO). Transwell clusters were purchased from Corning Costar (Cambridge, MA). Microsomes from baculovirus- infected insect cells expressing human CYP1A2, 2A6, 2B6, 2C8, 2C9*1(Arg 144), 2C19, 2D6*1, 2E1 and 3A4 and rat CYP 1A2, 2B1, 2C11, 2D1, 2E1, and 3A1 (Supersomes ${ }^{\mathrm{TM}}$ ) as well as insect cell control and rat P450 reductase insect cell control Supersomes ${ }^{\mathrm{TM}}$ were purchased from BD Gentest (Bedford, MA). The CYPs were co-expressed with their corresponding human or rat cytochrome P450 reductase, in addition, human CYP 2A6, 2B6, 2C8, 2C9*1, 2C19, 2E1, and 3A4 and rat CYP 2B1, 2C11, 2D1, 2E1, and 3A1 were co-expressed with human cytochrome b5. Pooled human liver microsomes, pooled male Sprague Dawley rat liver microsomes, and NADPH regenerating systems were also purchased from BD Gentest. Potassium monobasic phosphate and potassium dibasic phosphates were purchased from Fischer Scientific Co (Fair Lawn, NJ). The BZT analogs (Table 1) were synthesized as previously described (Newman et al., 1994; Newman et al., 1995; Kline et al., 1997; Katz 
et al., 2001). All chemicals and solvents were high-performance liquid chromatography (HPLC) grade or American Chemical Society analytical grade.

\section{METHODS}

\section{Cell Culture}

Cells were grown at $37^{\circ} \mathrm{C}, 95 \%$ relative humidity and $5 \% \mathrm{CO}_{2}$ atmosphere on 12-well Costar inserts (Transwell®; 0.4- $\mu \mathrm{m}$ pore polycarbonate filter, $1-\mathrm{cm}^{2}$ diameter). Caco-2 cells (passages 41 and 42 ) were seeded at a density of 80,000 cells $/ \mathrm{cm}^{2}$. The cells were grown for 26-27 days in Dulbecco's modified Eagle medium (1X), containing 10\% fetal bovine serum, $2 \%$ glutamine, $1 \%$ non essential amino acids, $1 \%$ penicillin- streptomycin, with the medium changed every other day. MDCK-MDR1 cells were seeded at a density of 425,000 cells $/ \mathrm{cm}^{2}$ and grown for 3-4 days in a medium similar to that used for Caco-2 cells with a daily medium change.

\section{Characterization of Caco-2 and MDCK-MDR1 Cell Monolayers}

Prior to conducting the transport experiments, monolayer integrity was determined by measuring the transepithelial electrical resistance (TEER) using a Millicell ${ }^{\circledR}$-ERS meter (Millipore Corp., Bedford, MA) and by determining the permeability of $\left[{ }^{14} \mathrm{C}\right]$ mannitol and $\left[{ }^{3} \mathrm{H}\right]$ propranolol. P-gp expression was functionally tested by conducting bidirectional transport studies with $\left[{ }^{14} \mathrm{C}\right]$ paclitaxel and determining its efflux ratio across Caco-2 and MDCK-MDR1 monolayers.

\section{BZT Analogs Bidirectional Transport and Inhibition Studies}

These studies were conducted to evaluate and compare the in-vitro permeability and the possible P-gp interaction of the four chloro BZT analogs (Table 1). The goal was to examine whether these analogs differ significantly in permeability and efflux in vitro. 
JPET \# 111245

Transport experiments for the chloro BZT analogs were performed in both the apical to basolateral (AB) and the basolateral to apical (BA) directions across Caco-2 and MDCKMDR1 monolayers in the presence and absence of the P-gp inhibitor verapamil (Wacher et al., 1995). At the time of experiment, the culture medium was removed from both the apical and basolateral sides of the monolayers and washed twice with PBS. The monolayers (n=3/gp) were incubated with either $200 \mu \mathrm{M}$ verapamil (Taub et al., 2005) in PBS or PBS for 30 minutes. Following the pre-incubation period, mixtures of the BZT analogue $(0.1 \mathrm{mM})$ with either verapamil $(200 \mu \mathrm{M})$ or with PBS were added to the donor compartments. The receiver compartments solution consisted of either $200 \mu \mathrm{M}$ verapamil in PBS (transport in presence of verapamil) or PBS (transport in absence of verapamil). For the apical to basolateral study, the inserts were moved to new transwells containing $1.5 \mathrm{ml}$ of the corresponding receiver compartment solution at $30,60,90$, and 120 minutes. For the basolateral to apical study, samples were drawn from the apical chamber at the same time points and replaced with equivalent volumes of fresh receiver compartment solution. Transport experiments were performed at $37^{\circ} \mathrm{C}$ with continuous agitation on a plate shaker $(75$ cycles $/ \mathrm{min})$ and samples were stored at $-80^{\circ} \mathrm{C}$ until the time of analysis.

\section{Characterization of Human and Rat Cytochrome P-450 Enzymes Involved in the Metabolism of 4,4“'- diCI BZT}

Since the four chloro analogs differ only in the position and number of chloro substitutions, we did not expect their in vitro metabolism to differ significantly. Consequently we selected 4`,4“-diCl BZT, as representative of the class, and screened for the enzymes involved in its metabolism. 4`,4“- $\mathrm{diCl}$ BZT was incubated with human 
CYP 1A2, 2A6, 2B6, 2C8, 2C9*1(Arg 144), 2C19, 2D6*1, 2E1 and 3A4 as well as rat CYP 1A2, 2B1, 2C11, 2D1, 2E1, and 3A1 Supersomes ${ }^{\mathrm{TM}}$ for 60 minutes. For each enzyme tested, the reaction mixture consisted of $50 \mathrm{pmol} / \mathrm{ml} \mathrm{CYP,} \mathrm{NADPH} \mathrm{regenerating}$ system (1.3 mM NADP+, $3.3 \mathrm{mM}$ glucose-6-phosphate, $0.4 \mathrm{U} / \mathrm{ml}$ glucose-6-phosphate dehydrogenase, and $3.3 \mathrm{mM}$ magnesium chloride), and $10 \mu \mathrm{M}$ of 4`,4“- diCl BZT in 100 $\mathrm{mM}$ potassium phosphate buffer $(\mathrm{pH}=7.4$, final volume $=500 \mu \mathrm{l})$. The reactions were initiated by adding ice cold Supersomes ${ }^{\mathrm{TM}}$ to the pre-warmed mixture of buffer, substrate and cofactors. After a $60 \mathrm{~min}$ incubation period at $37^{\circ} \mathrm{C}$, the reactions were stopped by the addition of $250 \mu 1$ acetonitrile and centrifuged $(10,000 \mathrm{x}$ g) for 5 minutes. Two hundred micro-liters of the supernatants were injected onto the HPLC for determination of unchanged 4`,4“-diCl BZT concentrations. Similar incubations with insect cell control and rat P450 reductase insect cell control Supersomes ${ }^{\mathrm{TM}}$ were performed to control for the native activities and non CYP specific effects. Metabolism incubations were performed in triplicates.

\section{Determination of the Time Course of 4,4“-diCl BZT Metabolism}

The time course of metabolism of 4 ", 4 " $-\mathrm{diCl}$ BZT ( $3 \mu \mathrm{M}$ final concentration, $\mathrm{n}=3$ ) by pooled human liver microsomes, pooled male rat liver microsomes and the human and rat CYPs that significantly reduced the concentration of 4 "4"-diCl BZT in the initial screening experiments was determined. The microsomes were used at a concentration of $0.8 \mathrm{mg} / \mathrm{ml}$. The CYP isoforms, cofactor, and buffer concentrations were similar as described above with a final reaction volume of $1500 \mu$. The reactions were initiated by adding the drug to the pre-warmed reaction mixture. After $0,5,10,20,30,40$, and 60 minutes of incubation at $37^{\circ} \mathrm{C}, 200 \mu \mathrm{l}$ of the reaction mixture were sampled, immediately 
vortexed with $100 \mu \mathrm{l}$ acetonitrile to terminate the reaction and centrifuged at $10,000 \mathrm{~g}$ for 5 minutes. Aliquots of the supernatant were then collected for HPLC analysis.

\section{Animal Pharmacokinetic Studies}

\section{Animals}

Adult male Sprague-Dawley rats (250-350 g) were used in the study and were purchased from Harlan (Indianapolis, IN). The animal study protocols were approved by the Institutional Animal Care and Use Committee of the School of Pharmacy (University of Maryland). Rats were housed in the animal facility at a room temperature of $72 \pm 2^{\circ} \mathrm{F}$, allowed free access to food (Purina 5001 Rodent Chow; Purina, St. Louis, MO) and water ad libitum and maintained on 12-h light/dark cycles.

\section{Pharmacokinetic Studies}

The rats were administered $44^{-} \mathrm{Cl} \mathrm{BZT}$ or $4,44^{\prime \prime}-\mathrm{diCl} \mathrm{BZT}$ at an i.v. dose of the free base equivalent to 5 or $10 \mathrm{mg} / \mathrm{kg}$ of the hydrochloride salt. $4-\mathrm{Cl}$ BZT was dissolved in sterile water for injection. 4,4“-diCl BZT was dissolved in 20\% (2-Hydroxypropyl)- $\beta$ cyclodextrin in sterile water for injection. The dosing for the pharmacokinetic studies was conducted at a volume of $1 \mathrm{ml} / \mathrm{kg}$. A destructive sampling design was adapted where groups of three animals were sacrificed by $\mathrm{CO}_{2}$ asphyxiation predose and postdose at 5 , $30,60,120,240,360,480,600$, and 1440 minutes. Blood was collected by heart puncture using heparinized syringes and centrifuged for 10 minutes at $3000 \mathrm{rpm}$. The plasma was separated and stored at $-80^{\circ} \mathrm{C}$ until the analysis. Brain tissues were immediately removed, blotted on a filter paper, weighted and stored at $-80^{\circ} \mathrm{C}$ until the time of analysis. 


\section{Analysis of the Transport, Metabolism and Pharmacokinetic Samples}

A previously published UV-HPLC method with few modifications was used for analysis of the BZT analogs (Raje et al., 2002). The chromatographic conditions consisted of a Symmetry ${ }^{\circledR} \mathrm{C}_{18}$ column $(150 \times 4.6 \mathrm{~mm}, 5 \mu \mathrm{m})$ for 4 4, 4 -diClBZT or a Supelcosil LC ABZ Plus column ( $250 \times 4.6 \mathrm{~mm}, 5 \mu \mathrm{m})$ (for the remaining BZT analogs), UV detector $(\lambda=220 \mathrm{~nm})$, mobile phases [methanol/0.05 $\mathrm{M} \mathrm{Na}_{2} \mathrm{HPO}_{4}(\mathrm{pH} 3.0), 40: 60(\mathrm{v} / \mathrm{v})(\mathrm{A})$ and methanol/0.05 M Na2HPO4 (pH 3.0), 80:20 (v/v) (B)], and a flow rate of $1 \mathrm{ml} / \mathrm{min}$ pumped using a gradient profile optimized for the different analogs and type of sample.

The transport and metabolism samples were analyzed directly without further processing. For the pharmacokinetic study, the plasma samples were extracted with hexane followed by evaporation and reconstitution in the mobile phase. The brain samples were double extracted with hexane following homogenization with distilled water. Oxprenolol and 4'$\mathrm{Cl}$ BZT were used as internal standards for 4`-Cl BZT and 4`,4“-diCl BZT respectively. The calibration curves were linear $\left(r^{2} \geq 0.994\right)$ in the range of 50 to $10,000 \mathrm{ng} / \mathrm{ml}, 100$ $10,000 \mathrm{ng} / \mathrm{ml}, 50-5000 \mathrm{ng} / \mathrm{ml}$, and $100-20,000 \mathrm{ng} / \mathrm{ml}$ for the transport, metabolism, plasma and brain matrices respectively. The radioactive samples of the transport markers were analyzed by addition to $5 \mathrm{ml}$ universol scintillation cocktail and measuring radioactivity using a Beckman Coulter LS 6500 Multi-purpose Scintillation Counter.

\section{Data Analysis}

\section{Transport Data Analysis}

The apparent permeability coefficients (in presence and absence of the P-gp inhibitor verapamil) were determined at sink conditions using the following equation:

$$
P_{a p p}=\frac{d Q / d t}{A^{*} C_{0}}
$$


where $\mathrm{dQ} / \mathrm{dt}$ is equal to the linear appearance rate of mass the receiver solution, $\mathrm{A}$ is the cross sectional area of the insert filters, and $\mathrm{C}_{0}$ is the donor concentration at time zero. All values are represented as mean and standard deviation from three transwell inserts. Efflux ratios across the monolayers were calculated using the equation:

$$
\text { Efflux Ratio }=\frac{P_{a p p}(B-A)}{P_{a p p}(A-B)}
$$

where $\mathrm{P}_{\mathrm{app}}(\mathrm{B}-\mathrm{A})$ is the permeability from the basolateral to the apical direction (secretory transport) and $\mathrm{P}_{\mathrm{app}}(\mathrm{A}-\mathrm{B})$ the permeability from the apical to basolateral direction (absorptive transport). Enhancement ratio in the apical to basolateral transport induced by verapamil was calculated according to the equation:

$$
\text { Enhancement Ratio }=\frac{P_{a p p}(A-B)_{v e r}}{P_{a p p}(A-B)}
$$

The statistical significance of effect of verapamil on the permeability of each of the BZT analogs studied was determined with two sample Student's t-test at $\alpha=0.05$ using Microsoft ${ }^{\circledR}$ Excel software. The delta method was used to calculate the standard error of the ratios and statistical significance was declared when the $95 \%$ confidence interval of two ratios did not overlap. Correlation analysis was conducted using SAS for windows software.

\section{Metabolism Data Analysis}

\section{Identification of the CYPs Involved in the Metabolism}

The human and rat CYP isoforms involved in the metabolism of 4,4"'-di Cl BZT were identified by analyzing the differences in mean substrate concentrations remaining after 60 min incubations by one-way analysis of variance (ANOVA) followed by Dunnett's multiple comparisons of CYP incubations versus control incubations. We also calculated 
the $\%$ of the mean control concentration remaining after 60 min incubation according to the equation:

$$
\% \text { of substrate remaining after } 60 \mathrm{~min}=\frac{C_{C Y P r, 60 \mathrm{~min}}}{C_{\text {average } \mathrm{ctrl}, 60 \mathrm{~min}}} * 100
$$

where $\mathrm{C}_{\mathrm{CYPr}, 60 \mathrm{~min}}$ is the substrate concentration from the $\mathrm{r}^{\text {th }}$ replicate after 60 min of incubation with a particular CYP Supersome ${ }^{\mathrm{TM}}$, and $\mathrm{C}_{\text {average }}$ ctrl, 60 min is the average $(\mathrm{n}=3)$ substrate concentration after 60 min incubation with insect cell control Supersomes ${ }^{\mathrm{TM}}$ (for human CYPs) or rat P450 reductase insect cell control Supersomes ${ }^{\mathrm{TM}}$ (for rat CYPs). The percentages are represented as mean and standard deviation from triplicate reactions.

\section{Intrinsic Clearance Calculation}

The intrinsic clearance values were calculated based on the substrate disappearance rate as previously described (Naritomi et al., 2001; Naritomi et al., 2003). Assuming first order disappearance of substrate, the disappearance rate constant $\left(\mathrm{K}_{\mathrm{e}}\right)$ was calculated from the slope of $\log \mathrm{C}_{\mathrm{t}}$ versus time profile based on the equation:

$$
\log C_{t}=\log C_{o}-\frac{K_{e} * t}{2.303}
$$

where $C_{t}$ is the concentration of the substrate at the different time points, $C_{0}$ is the substrate concentration at time zero. The initial metabolic rate $\left(\mathrm{V}_{0}\right)(\mathrm{pmol} / \mathrm{min} / \mathrm{pmol} \mathrm{CYP}$ or mg microsomal protein) was calculated from the equation:

$$
V_{0}=\frac{K_{e} * C_{0}}{P_{M S}}
$$

where $\mathrm{P}_{\mathrm{MS}}$ is CYP concentration ( $\left.\mathrm{pmol} / \mathrm{ml}\right)$ or microsomal protein concentration $(\mathrm{mg} / \mathrm{ml})$. $\mathrm{V}_{0}$ can also be described using Michaelis-Menten equation as follows: 


$$
V_{0}=\frac{V \max * C_{0}}{K m+C_{0}}
$$

Assuming that $\mathrm{C}_{0} \ll<\mathrm{Km}$, equation (7) can be written as:

$$
V_{0}=\frac{V_{\max } * C_{0}}{K m}
$$

Accordingly, the intrinsic clearance was calculated based on the formula:

$$
C L_{\text {int }}=\frac{V \max }{K m}=\frac{V_{0}}{C_{0}}
$$

The intrinsic clearance values were calculated separately from each of the replicates and compared statistically using one way ANOVA followed by Duncan multiple range test. $\mathrm{CL}_{\text {int }}$ values are presented as mean $\pm \mathrm{SD}$ from the three replicates performed for each reaction.

\section{Pharmacokinetic Data Analysis}

The destructive sampling data obtained from the pharmacokinetic studies were initially analyzed by the naïve averaging method. For a given drug, the plasma concentrations from the three animals at each time point for each dose level were averaged. The average concentrations versus time data were then used for compartmental modeling using WinNonlin version 4.1 software (Pharsight, Cary, NC). Various compartment models were evaluated to determine the most appropriate model. In addition, various weighting schemes including equal weight, $1 / \mathrm{y}, 1 / \hat{y}, 1 / \mathrm{y}^{2}$ and $1 / \hat{y}^{2}$ were evaluated where $\mathrm{y}$ is the observed drug concentration and $\hat{y}$ is the model predicted drug concentration. The final model selection was based on the visual inspection of goodness of fit plots and on the value of WSSR, precision of parameter estimates, Akaike's information criteria and Schwarz criteria. 
JPET \# 111245

To be able to estimate the inter-animal variability in the pharmacokinetic parameters, non linear mixed effect modeling was conducted using NONMEM version 5, level 1.1 (GloboMax LLC, Hanover, MD). For each drug, the data from the two dose levels were compiled and analyzed (Data from 42 animals for 4`-Cl BZT and 54 animals for 4,4“-diClBZT). The final parameter estimates from the naïve averaging analysis were used as initial estimates for the population analysis. Based on the results from the naïve averaging analysis, the population analysis started with a two compartment structural model for both $4-\mathrm{Cl}$ BZT and 4,4“-diCl BZT. Additive, proportional and exponential statistical error models were evaluated to describe the inter-animal as well as the residual variability. The analysis was performed using the conventional first order estimation method. The final model was determined based on inspection of goodness of fit plots, precision of parameter estimates and the value of the objective function. In addition, the Likelihood Ratio Test (at $\alpha=0.05$ ) was used as a selection criterion between rival hierarchical models (Sheiner and Ludden, 1992). The brain to plasma partition coefficient $\left(\mathrm{R}_{\mathrm{i}}\right)$ was calculated as a measure of brain uptake according to the formula:

$$
R i=\frac{\mathrm{AUC}_{0}-\inf (\text { brain })}{\mathrm{AUC}_{0}-\inf \text { (plasma) }}
$$

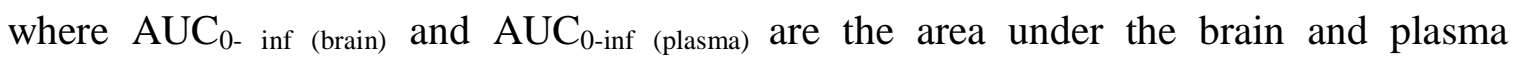
concentrations versus time curves, respectively. The $\mathrm{AUC}_{0 \text {-inf }}$ were calculated using WinNonlin non-compartmental analysis by applying the linear trapezoidal rule for the ascending portions of the concentration versus time profiles and the log linear trapezoidal rule for the descending portions. For each dose, the pharmacokinetic parameters for $4{ }^{`}-\mathrm{Cl}$ BZT and 4,4“-diCl BZT were compared using a two sample Student t-test. The pharmacokinetic parameters for the $5 \mathrm{mg} / \mathrm{kg}$ dose for the studied analogs were compared 
to previously published parameters for cocaine at the same dose (Raje et al., 2003) using one way ANOVA followed by Dunnett's post hoc analysis. 
JPET \# 111245

\section{RESULTS}

\section{Characterization of the Cell Lines}

Across MDCK-MDR1 monolayers, mannitol $\mathrm{P}_{\text {app }}$ ranged from 3.89 to $4.46 \times 10^{-6}$ $\mathrm{cm} / \mathrm{sec}$ and the TEER values were $>600 \Omega . \mathrm{cm}^{2}$. Propranolol $\mathrm{P}_{\text {app }}$ was $24.33 \times 10^{-6} \mathrm{~cm} / \mathrm{sec}$ and paclitaxel efflux ratio was 101 indicating high level of P-gp expression. Across Caco2 monolayers, mannitol $\mathrm{P}_{\text {app }}$ ranged from 0.22 to $0.28 \times 10^{-6} \mathrm{~cm} / \mathrm{sec}$ and the TEER values ranged from 450 to $500 \Omega . \mathrm{cm}^{2}$ indicating high monolayer integrity. Propranolol permeability was $13.34 \times 10^{-6} \mathrm{~cm} / \mathrm{sec}$ indicating higher transcellular resistance when compared to MDCK-MDR1 cells. Paclitaxel efflux ratio ranged from 40 to 43 across Caco-2 cells.

\section{MDCK-MDR1 Chloro BZT analogs Permeability}

Across MDCK-MDR1 monolayers, the chloro analogs have shown permeability values ranging from 8.26 to $32.23 \times 10^{-6} \mathrm{~cm} / \mathrm{sec}$ with the following rank order: $3 \mathrm{Cl} \mathrm{BZT}$

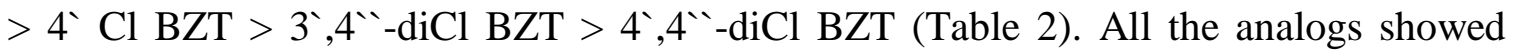
polarized transport with higher basolateral to apical (B-A) permeability than the apical to basolateral (A-B) permeability. The efflux ratio ranged from 2.1 to 6.9 with the highest efflux observed with 4,4" - $\mathrm{diCl}$ BZT and the lowest with the mono substituted chloro analogs (Table 2).

\section{Caco-2 BZT Permeability}

The studied analogs displayed polarized transport across Caco-2 monolayers also. The permeability values ranged from 1.37 to $21.65 \times 10^{-6} \mathrm{~cm} / \mathrm{sec}$ with rank order similar to that observed with MDCK-MDR1 cells, i.e: $3 `-\mathrm{Cl}$ BZT > 4`-Cl BZT > 3`,4``-diCl BZT > 4,4"-diCl BZT (Table2). The efflux ratio ranged from 3.3 to 28.4 with the 
JPET \# 111245

highest efflux observed with 4`4“-di Cl BZT replicating the efflux pattern observed with the MDCK-MDR1 cells (Table 2).

\section{Effect of Verapamil on BZT Polarized Transport}

Verapamil was used to verify the involvement of P-gp in mediating the observed efflux of the BZT analogs. Verapamil increased the A-B permeability of the BZT analogs in both MDCK-MDR1 and Caco-2 cell lines (Figures 2A and 2B, respectively). The increase in the A-B permeability was statistically significant $(\mathrm{p}<0.05)$ for all the analogs except for $3 `-\mathrm{Cl}$ BZT (Figures 2A and 2B) where statistical significance was not reached; probably due to the high variability. The enhancement ratio of the A-B transport induced by verapamil ranged from 1.1 for $3{ }^{`}-\mathrm{Cl}$ BZT to 3.2 for 4 ',4'- diCl BZT across MDCKMDR1 monolayers (Figure 2A) and from 1.3 for $3{ }^{`}-\mathrm{Cl}$ BZT to 4.3 for $44^{`}, 4^{`}{ }^{-}$-di Cl BZT across Caco-2 monolayers (Figure 2B). Except for $3{ }^{`}-\mathrm{Cl}$ BZT in MDCK-MDR1 cells, verapamil reduced the efflux ratios of all the BZT analogs across both cell lines and the reduction was statistically significant in most instances (Figures 2C and 2D). At $200 \mu \mathrm{M}$, MTS and XTT cytotoxicity assays indicated that verapamil did not affect either MDCKMDR1 or Caco-2 cell viability $(\mathrm{p}<0.05)$ over 2.5 hours of exposure at conditions identical to those in which the transport experiments were conducted (data not shown).

\section{Characterization of Human and Rat Cytochrome P-450 Enzymes Involved in the Metabolism of 4',4”-diCl BZT}

The objective of these studies was to screen for the enzymes involved in the metabolism of $4{ }^{\prime}, 4$ " $-\mathrm{diCl} \mathrm{BZT}$ as a compound representative of the class. Among the CYP isoforms tested, human CYP 2C19, CYP 2D6, and rat CYP 2 C11 resulted in statistically significant disappearance of 4`,4“-diCl BZT after 60 minute incubation when 
compared to the control $(\mathrm{p}<0.05)$ (Figure 3 A and 3 B). Based on the post hoc analysis, the effect of rat CYP 3A1 was not statistically significant although a t-test comparison to the control have shown $\mathrm{p}$ value less than 0.05 . The most extensive substrate disappearance was observed with 4’,4“-diCl BZT using rat CYP 2C11 (6.05 $\pm 0.26 \%$ of the parent compound remaining at the end of incubation period) (Figure 3B).

\section{Intrinsic Clearance of 4,4“-di Cl BZT in Human and Rat Recombinant CYPs and Pooled Human and Male Rat Liver Microsomes}

Under the experimental conditions, 4,4“-di Cl BZT metabolism followed first order reaction with the concentration of the substrate declining mono-exponentially with time. The calculated intrinsic clearance values of 4,4“-diCl BZT are reported in Table 3. The preference for metabolizing 4`4“-diCl BZT was as follows CYP 2C19 > CYP 2D6 for human CYPs and CYP 2C11 > CYP 3A1 for rat CYPs. The differences in the intrinsic clearance between human CYP 2D6, human CYP 2C19 and rat CYP 3A1 were not statistically significant as reported in Table 3. The intrinsic clearance of 4 '4“-diCl BZT was 1.68 fold higher in pooled male rat liver microsomes than in human liver microsomes.

\section{Pharmacokinetics of 4'-Cl and 4,4”'-diCl BZT}

Two analogs were selected for the pharmacokinetics and brain distribution study as they represent the two extremes among the chloro BZT analogs in terms of their behavioral profiles (Katz et al. 1999). In addition, they differed significantly in their in vitro transport and their P-gp mediated efflux. Figure 4 A represents the observed and the predicted plasma concentrations of $4{ }^{\prime}-\mathrm{Cl}$ BZT and 4,4“-diCl BZT upon i.v. administration to male Sprague Dawely rats at two dose levels ( 5 and $10 \mathrm{mg} / \mathrm{kg}$ ) based 
on the best fit achieved with WinNonlin. Figure 4B represents the corresponding brain concentrations versus time profiles. The brain concentrations were higher than the plasma concentrations at each time point. The pharmacokinetics of 4`-Cl BZT and 4`4“--diCl BZT were best described with a two compartmental model. The final parameter estimates from WinNonlin were used as initial estimates for the population analysis. The final population model consisted of a two compartmental structural model parameterized in terms of clearance (CL), volume of the central compartment $(\mathrm{Vc})$, volume of the peripheral compartment (Vp) and inter-compartmental clearance (Q) (ADVAN3 TRANS4 NONMEM Subroutines). The inter-animal variability in Vc, Vp and Q for 4$\mathrm{Cl}$ BZT and in CL, Vc, and Q for 4",4"-diCl BZT were best described with an exponential pharmacostatistical model. Based on the Likelihood Ratio Test, the interanimal variability in $\mathrm{CL}$ for 4 - $\mathrm{Cl}$ BZT and Vp for 4`,4“-diCl BZT were not significantly different from zero $(\mathrm{p}>0.05)$. Consequently, they were omitted from the final model. The residual random error was best described with a proportional model. The residual error was estimated to be $13.4 \%$ for $4 `-\mathrm{Cl}$ BZT and was fixed to $11.8 \%$ for 4 ‘, 4 “-diCl BZT based on a sensitivity analysis and the value selected was the one resulting in the lowest objective function and the highest precision in the estimation of the inter-animal variability.

Figure 5 represents the relevant diagnostic plots for the final population models for 4 - $-\mathrm{Cl}$ BZT and 4",4"-diCl BZT and indicates a lack of any systematic bias in describing the pharmacokinetic profiles. The population pharmacokinetic parameters along with their associated inter-animal variability and the precision of the parameter estimates (expressed as coefficient of variation) are reported in Table 4 . The secondary pharmacokinetic 
parameters of interest along with their associated standard deviation were calculated from the post hoc estimates of the individual animal parameters provided by NONMEM and are reported in Table 5. Data obtained after a single $5 \mathrm{mg} / \mathrm{kg}$ i.v. dose of cocaine to rats are presented for comparison in Table 5 (Raje et al., 2003). Both 4`-Cl BZT and 4`,4“diCl BZT had significantly longer half-life $\left(\mathrm{t}_{1 / 2}\right)$ and larger steady state volume of distribution (Vss) when compared to cocaine at the same dose $(\mathrm{p}<0.05)$. When comparing the two BZT analogs across the same doses, the elimination half life of 4 ,4"diCl BZT was significantly longer than 4-Cl BZT $(\mathrm{p}<0.001)$. The Vss for 4`,4“-diCl BZT was significantly lower than that of 4`-Cl BZT $(\mathrm{p}<0.001)$. The difference in steady state volume of distribution resulted mainly from the difference in the volume of the peripheral compartment (Table 4). For a given analog, the effect of the dose on the Vss and $t_{1 / 2}$ was not statistically significant except for $4{ }^{\prime}, 4$ “-diCl BZT where the $t_{1 / 2}$ for the $10 \mathrm{mg} / \mathrm{kg}$ dose was significantly longer than the $5 \mathrm{mg} / \mathrm{kg}$ dose $(\mathrm{p}<0.05)$. Based on the results of the non-compatmental analysis, the brain uptake for 4`-Cl BZT and 4`4“'-diCl BZT seemed to be comparable and higher than cocaine (Table 5). In addition, the brain $t_{1 / 2}$ for each BZT analog was considerably longer than cocaine (Table 5). In general, the pharmacokinetics of the two BZT analogs tested seems to be almost linear over the dose range studied (Table 5 and Figure 4). 
JPET \# 111245

\section{DISCUSSION}

Several reports have indicated that the benztropine analogs possess behavioral profiles that are significantly different from cocaine despite their high in vitro affinity and selectivity to DAT and their high potency as DA uptake inhibitors. However, not all benztropine analogs are equally devoid of cocaine like effects, but rather they produce dissimilar behavioral profiles. These behavoiral differences are not necessarily explainable by the binding and affinity profiles at DAT. Consequently, several hypotheses have been suggested to explain such differences (Katz et al., 2001; Woolverton et al., 2001; Desai et al., 2005; Li et al., 2005).

It is well recognized that the rate and extent of brain uptake and consequently DAT occupancy are important determinants of the behavioral profiles and the abuse liability of the DA uptake inhibitors. Rapid brain uptake and fast association with DAT as well as a certain level of DAT occupancy has been predicted to cause high abuse liability (Volkow et al., 1997; Gorelick, 1998). In addition, the short duration of action of some drugs of abuse (e.g., cocaine) is believed to contribute to the craving and the need for repeated administration (Quinn et al., 1997). Consequently, the present studies were conducted to delineate the contribution of dispositional factors in mediating the behavioral differences of the chloro BZT analogs.

The chloro BZT analogs are basic lipophilic molecules that contain oxygen and nitrogen atoms which can function as hydrogen bond acceptors. These characteristics suggest possible interaction with the efflux transporter P-gp, which may modulate their brain uptake. In the present study, the BZT analogs have shown polarized transport across the P-gp over-expressing MDCK-MDR1 cells and Caco-2 cells; in agreement with 
the apical localization of P-gp. The efflux ratios of the chloro analogs were in the reverse order of their permeability values with 4^4“-diCl BZT having noticeably high efflux followed by $3{ }^{\prime}, 4$ “-diCl BZT followed by $4 `-\mathrm{Cl}$ and $3 `-\mathrm{Cl}$ BZT. The higher efflux of the di-chloro analogs mediated their reduced in vitro permeability and may be a result of their higher lipophilicity when compared to the mono-substituted analogs (Table 1). In addition, by examining the efflux ratios of the chloro analogs, it seems that the para chloro substitution enhances the affinity of the BZT analogs to P-gp over the meta substitution. Interestingly, the in vitro permeability and efflux data correlated well with the reported behavioral differences aforementioned in the introduction section.

The P-gp inhibitor verapamil enhanced the A-B permeability and reduced the efflux ratios of the chloro BZT analogs verifying that P-gp was responsible for the observed efflux. However, verapamil did not totally abolish it. Taking into account that verapamil inhibits P-gp competitively; the attenuated efflux in the presence of verapamil may be a result of the relatively high concentration of the BZT analogs used in the experiments $(100 \mu \mathrm{M})$ or due to high affinity of these analogs for P-gp.

In vivo, 4`4“-diCl BZT, in spite of its higher in vitro P-gp efflux, showed a comparable brain to plasma partition coefficient to $4{ }^{`} \mathrm{Cl}$ BZT. The higher lipophilicity of 4`,4“-diCl BZT (Table 1) may have resulted in offsetting the effect of its higher P-gp efflux, resulting in comparable brain uptake to $4^{-}-\mathrm{Cl}$ BZT. Similar pattern was previously observed with the BZT analog JHW 007 (N-(n-butyl)-(bis-fluorophenyl)methoxytropane , cLogP= 5.53) (Raje et al., 2003). JHW007 had high brain uptake $(\mathrm{Ri}=5.6)$ despite its high observed P-gp efflux (efflux ratio of 8 across BBMEC monoalyers). However, the volume of the peripheral compartment was lower for 4`,4“-diCl BZT in comparison to 
JPET \# 111245

4-Cl BZT which could be a result of P-gp affecting the distribution of 4,4“-diCl BZT to other tissues. Overall, the higher brain uptake of the two chloro analogs in comparison to cocaine excludes the possibility that limited extent of permeation across the BBB is responsible for their lack of cocaine like effects or for their behavioral differences (compared to one another). In addition, it indicates that P-gp plays a very minor role, if any, in limiting the extent of $\mathrm{BBB}$ transport of this class of compounds. However, it should be noted that the time resolution of our sampling technique did not allow us to capture the entry phase to the brain, as the brain $\mathrm{C}_{\max }$ was observed at the first sampling time ( 5 minutes post dose). Consequently, we were not able to determine whether these two analogs differ from one another and from cocaine in their rate of brain entry within the first five minutes of exposure and if P-gp has any role in modifying that rate. In addition, our pharmacokinetic studies were conducted via i.v. drug administration while the behavioral evaluations, except for the self administration procedures, where conducted using the i.p. route. Consequently, the possibility that these analogs could differ in their rate or extent of absorption from the peritoneal cavity should not be discounted.

The pharmacokinetic study also indicated that 4`-Cl BZT and 4 ,4“-diCl BZT are characterized by larger Vss when compared to cocaine (Vss rank order: 4`Cl BZT > 4,4“-diCl BZT>>> cocaine). This is partially because of the higher brain uptake for the BZT analogs. Both 4'-Cl BZT and 4,4“'-diCl BZT are characterized by longer elimination $\mathrm{t}_{1 / 2}$ when compared to cocaine $\left(\mathrm{t}_{1 / 2}\right.$ rank order: $44^{\prime}, 4^{\prime}{ }^{\prime}-\mathrm{diCl} \mathrm{BZT}>>4^{`}-\mathrm{Cl}$ BZT >>cocaine). Cocaine undergoes rapid non enzymatic as well as enzymatic hydrolysis by the plasma and liver esterases and this is responsible for its short duration 
JPET \# 111245

of action (Quinn et al., 1997). The long $\mathrm{t}_{1 / 2}$ relative to cocaine explains the previously reported prolonged duration of stimulation of locomotor activity caused by the chloro BZT analogs when compared to cocaine (Katz et al., 1999; Katz et al., 2001) as well as the prolonged elevation of brain dopamine level caused by $4-\mathrm{Cl}$ BZT in comparison to cocaine (Tanda et al., 2005).

The clearance of 4`,4”-diCl BZT from the brain was slower than 4`-Cl BZT which was much slower than cocaine as can be seen from the brain half lives (Table 5). The high brain uptake and the slow clearance coupled with the high affinity of the chloro analogs to DAT could result in saturation of DAT for a long period of time upon administration of the first few doses. Consequently, this will interfere with the frequent dosing in the self administration procedures since any subsequent doses of these analogs will not be as rewarding due to the possible DAT saturation. 4`,4“-diCl BZT was not evaluated in self administration studies but assuming the similarity in the pharmacokinetic profile between $3{ }^{\prime}, 4{ }^{\prime}-\mathrm{diCl} \mathrm{BZT}$ and $4{ }^{\prime}, 4^{\prime}-\mathrm{diCl} \mathrm{BZT}$, due to the minimal structural difference, the difference in the brain clearance may explain the rank order for reinforcing effectiveness observed in self administration studies in rhesus monkeys (cocaine $>3^{`}-\mathrm{Cl} \mathrm{BZT}=4^{`}-\mathrm{Cl} \mathrm{BZT}>>3^{`}, 4^{`}{ }^{\prime}-\mathrm{diCl} \mathrm{BZT}$ ) as previously reported (Woolverton et al., 2001).

Although benztropine (Cogentin) has been on the market for many years, the enzymes involved in its metabolism have not been characterized. Accordingly, little is known about the metabolism of its analogs. In general, the structure and the pharmacokinetic data suggest higher metabolic stability for the BZT analogs in comparison to cocaine. The results of our metabolism study indicate that the human and 
rat CYP 2D and CYP 2C subfamilies are probably the key players in the metabolism of the BZT analogs. The CYP screening indicated that 4,4 " - $\mathrm{diCl}$ BZT is a substrate of human CYP 2D6. This is in agreement with the clinical reports which indicated that CYP 2D6 inhibitors augment the side effects of benztropine and increased its serum levels (Roth et al., 1994; Armstrong and Schweitzer, 1997). 4,4“"-diCl BZT is also a substrate for CYP 2C19 with intrinsic clearance comparable to CYP 2D6 (Table 3). The rat CYP screening indicated that 4,4“-diCl BZT is a substrate for CYP 2C11 and CYP 3A1 (with CYP 2C11 resulting in the most extensive metabolism among the entire panel tested). Our data also indicate that the clearance of $4,4{ }^{\prime \prime}-\mathrm{diCl} \mathrm{BZT}$ is faster in pooled rat liver microsomes than in pooled human liver microsomes. This may be indicative of longer duration of action in humans as compared to rats which may result in even lower abuse potential in humans. The validity of the last conclusion will depend on the fraction metabolized versus excreted unchanged and how these in-vitro data will extrapolate to the in-vivo situation.

In conclusion, the results presented in this paper indicate that the chloro BZT analogs possess high and comparable brain uptake despite their differences in lipophilicity and in interaction with P-gp. In addition, there are significant differences among the chloro analogs in clearance and duration of action. These differences may explain some aspects of the behavioral differences observed among the chloro BZT analogs and between them and cocaine. The present results also provide the first report on the enzymes involved in the metabolism of the BZT analogs. This will help in predicting the possible drug-drug interactions and can be regarded as the initial step to understanding the metabolic profile of the BZT analogs; a series of compounds to which 
JPET \# 111245

extensive research has been recently devoted to and from which a successful cocaine therapeutic may come. 
JPET \# 111245

\section{ACKNOWLEDGEMENTS}

The authors are grateful to Dr. Jonathan Katz for his critical reading of an earlier version of this manuscript. We also acknowledge Dr. Santosh Kulkarni and Ms. J. Cao for synthesizing multi-gram quantities of the BZT analogs used in this study. 
JPET \# 111245

\section{REFERENCES}

Armstrong SC and Schweitzer SM (1997) Delirium associated with paroxetine and benztropine combination. Am J Psychiatry 154:581-582.

Carroll FI, Howell LL and Kuhar MJ (1999) Pharmacotherapies for treatment of cocaine abuse: preclinical aspects. J Med Chem 42:2721-2736.

Desai RI, Kopajtic TA, Koffarnus M, Newman AH and Katz JL (2005) Identification of a dopamine transporter ligand that blocks the stimulant effects of cocaine. $J$ Neurosci 25:1889-1893.

Gorelick DA (1998) The rate hypothesis and agonist substitution approaches to cocaine abuse treatment. Adv Pharmacol 42:995-997.

He H, McKay G and Midha KK (1995) Phase I and II metabolites of benztropine in rat urine and bile. Xenobiotica 25:857-872.

Katz JL, Agoston GE, Alling KL, Kline RH, Forster MJ, Woolverton WL, Kopajtic TA and Newman AH (2001) Dopamine transporter binding without cocaine-like behavioral effects: synthesis and evaluation of benztropine analogs alone and in combination with cocaine in rodents. Psychopharmacology (Berl) 154:362-374.

Katz JL, Izenwasser S, Kline RH, Allen AC and Newman AH (1999) Novel 3alphadiphenylmethoxytropane analogs: selective dopamine uptake inhibitors with behavioral effects distinct from those of cocaine. J Pharmacol Exp Ther 288:302315. 
Katz JL, Kopajtic TA, Agoston GE and Newman AH (2004) Effects of N-substituted analogs of benztropine: diminished cocaine-like effects in dopamine transporter ligands. J Pharmacol Exp Ther 309:650-660.

Kline RH, Izenwasser S, Katz JL, Joseph DB, Bowen WD and Newman AH (1997) 3'Chloro-3 alpha-(diphenylmethoxy)tropane but not 4'-chloro-3 alpha(diphenylmethoxy)tropane produces a cocaine-like behavioral profile. $J$ Med Chem 40:851-857.

Kulkarni SS, Grundt P, Kopajtic T, Katz JL and Newman AH (2004) Structure-activity relationships at monoamine transporters for a series of N-substituted 3alpha(bis[4-fluorophenyl]methoxy)tropanes: comparative molecular field analysis, synthesis, and pharmacological evaluation. J Med Chem 47:3388-3398.

Li SM, Newman AH and Katz JL (2005) Place Conditioning and Locomotor Effects of N-Substituted, 4',4"-Difluorobenztropine Analogues in Rats. J Pharmacol Exp Ther.

Naritomi Y, Terashita S, Kagayama A and Sugiyama Y (2003) Utility of hepatocytes in predicting drug metabolism: comparison of hepatic intrinsic clearance in rats and humans in vivo and in vitro. Drug Metab Dispos 31:580-588.

Naritomi Y, Terashita S, Kimura S, Suzuki A, Kagayama A and Sugiyama Y (2001) Prediction of human hepatic clearance from in vivo animal experiments and in vitro metabolic studies with liver microsomes from animals and humans. Drug Metab Dispos 29:1316-1324. 
Newman AH, Allen AC, Izenwasser S and Katz JL (1994) Novel 3 alpha(diphenylmethoxy)tropane analogs: potent dopamine uptake inhibitors without cocaine-like behavioral profiles. J Med Chem 37:2258-2261.

Newman AH, Kline RH, Allen AC, Izenwasser S, George C and Katz JL (1995) Novel 4'-substituted and 4',4"-disubstituted 3 alpha-(diphenylmethoxy)tropane analogs as potent and selective dopamine uptake inhibitors. $J$ Med Chem 38:3933-3940.

Newman AH and Kulkarni S (2002) Probes for the dopamine transporter: new leads toward a cocaine-abuse therapeutic--A focus on analogues of benztropine and rimcazole. Med Res Rev 22:429-464.

Quinn DI, Wodak A and Day RO (1997) Pharmacokinetic and pharmacodynamic principles of illicit drug use and treatment of illicit drug users. Clin Pharmacokinet 33:344-400.

Raje S, Cao J, Newman AH, Gao H and Eddington ND (2003) Evaluation of the bloodbrain barrier transport, population pharmacokinetics, and brain distribution of benztropine analogs and cocaine using in vitro and in vivo techniques. $J$ Pharmacol Exp Ther 307:801-808.

Raje S, Dowling TC and Eddington ND (2002) Determination of the benztropine analog AHN-1055, a dopamine uptake inhibitor, in rat plasma and brain by highperformance liquid chromatography with ultraviolet absorbance detection. $J$ Chromatogr B Analyt Technol Biomed Life Sci 768:305-313.

Ritz MC, Lamb RJ, Goldberg SR and Kuhar MJ (1987) Cocaine receptors on dopamine transporters are related to self-administration of cocaine. Science 237:1219-1223. 
Roth A, Akyol S and Nelson JC (1994) Delirium associated with the combination of a neuroleptic, an SSRI, and benztropine. J Clin Psychiatry 55:492-495.

Rothman RB (1990) High affinity dopamine reuptake inhibitors as potential cocaine antagonists: a strategy for drug development. Life Sci 46:PL17-21.

Rothman RB, Blough BE, Woolverton WL, Anderson KG, Negus SS, Mello NK, Roth BL and Baumann MH (2005) Development of a rationally designed, low abuse potential, biogenic amine releaser that suppresses cocaine self-administration. $J$ Pharmacol Exp Ther 313:1361-1369.

Sheiner LB and Ludden TM (1992) Population pharmacokinetics/dynamics. Annu Rev Pharmacol Toxicol 32:185-209.

Tanda G, Ebbs A, Newman AH and Katz JL (2005) Effects of 4'-Chloro-3\{alpha $\}$ (diphenylmethoxy)-tropane on Mesostriatal, Mesocortical, and Mesolimbic Dopamine Transmission: Comparison with Effects of Cocaine. J Pharmacol Exp Ther 313:613-620.

Taub ME, Podila L, Ely D and Almeida I (2005) Functional assessment of multiple Pglycoprotein (P-gp) probe substrates: influence of cell line and modulator concentration on P-gp activity. Drug Metab Dispos 33:1679-1687.

Volkow ND, Wang GJ, Fischman MW, Foltin RW, Fowler JS, Abumrad NN, Vitkun S, Logan J, Gatley SJ, Pappas N, Hitzemann R and Shea CE (1997) Relationship between subjective effects of cocaine and dopamine transporter occupancy. Nature 386:827-830. 
Wacher VJ, Wu CY and Benet LZ (1995) Overlapping substrate specificities and tissue distribution of cytochrome P450 3A and P-glycoprotein: implications for drug delivery and activity in cancer chemotherapy. Mol Carcinog 13:129-134.

Woolverton WL, Hecht GS, Agoston GE, Katz JL and Newman AH (2001) Further studies of the reinforcing effects of benztropine analogs in rhesus monkeys. Psychopharmacology (Berl) 154:375-382.

Woolverton WL, Rowlett JK, Wilcox KM, Paul IA, Kline RH, Newman AH and Katz JL (2000) 3'- and 4'-chloro-substituted analogs of benztropine: intravenous selfadministration and in vitro radioligand binding studies in rhesus monkeys. Psychopharmacology (Berl) 147:426-435.

Zou MF, Kopajtic T, Katz JL and Newman AH (2003) Structure-activity relationship comparison of (S)-2beta-substituted 3alpha-(bis[4fluorophenyl]methoxy)tropanes and (R)-2beta-substituted 3beta-(3,4dichlorophenyl)tropanes at the dopamine transporter. J Med Chem 46:2908-2916. 
JPET \# 111245

\section{FOOTNOTES}

This work was supported by the NIH grant R01 DA16715-03 from the National Institute of Drug Abuse - Intramural Research Program (NIDA-IRP). 
JPET \# 111245

\section{LEGENDS FOR FIGURES}

Figure 1. Chemical structure of the BZT analogs studied. The chloro substituents (R`and $\left.\mathrm{R}^{\prime \prime}\right)$ are presented in Table 1.

Figure 2. Effect of verapamil $(200 \mu \mathrm{M})$ on permeability and P-gp mediated efflux of the chloro BZT analogs across MDCK-MDR1 and Caco-2 monolayers. (A) and (B): Apical to basolateral permeability in absence and in presence of verapamil across MDCKMDR1 and Caco-2 monolayers respectively (data represented as mean \pm SD). (C) and (D): efflux ratios across MDCK-MDR1 and Caco-2 monolayers in absence and in presence of verapamil (data represented as the ratio of the mean permeability values \pm SEM calculated using delta method). ${ }^{*} \mathrm{p}<0.05$. Black bar: in absence of verapamil. Grey bar: in presence of verapamil.

Figure 3. Metabolism of 4',4' '-diCl BZT (a representative analog of the class) by the human recombinant CYPs (A) and the rat recombinant CYPs (B). 4',4',-diCl BZT (10 $\mu \mathrm{M})$ was incubated with the supersomes $(50 \mathrm{pmol} / \mathrm{ml})$ and cofactors for $60 \mathrm{~min}$ followed by termination of reactions and determination of unchanged substrate. Data are presented as mean percentage of the average control $\pm \mathrm{SD}$ from triplicate reactions. $* \mathrm{p}<.0 .05$ based on Dunnett's test.

Figure 4. Plasma and brain pharmacokinetic profiles of 4`-Cl BZT and 4`4``-diCl BZT (5 and $10 \mathrm{mg} / \mathrm{kg}$ i.v.) in male Sprague-Dawley Rats ( $\mathrm{n}=3 /$ time point/dose). A: observed (mean $\pm \mathrm{SD}$ ) and predicted plasma concentration versus time profiles based on the naïve averaging analysis conducted using WinNonlin. B: Observed brain concentrations (mean \pm SD) versus time profiles. 
Figure 5. Diagnostic plots for the population analysis of the plasma concentrations of 4'ClBZT ( $\mathrm{n}=42$ rats) and 4`,4“-diCl BZT (n=54 rats) conducted using NONMEM.

For each drug, the data from the two dose levels tested ( $5 \mathrm{mg} / \mathrm{kg}$ i.v. and $10 \mathrm{mg} / \mathrm{kg}$ i.v.) were compiled prior to the analysis. A: population predicted versus observed plasma concentrations. B: individual animal predicted versus observed plasma concentrations. C: weighted residuals versus population predicted plasma concentrations. 
Table 1. Substitutions, cLog P, binding affinity at DAT of the BZT analogs studied as well as DA uptake inhibition by these analogs

\begin{tabular}{|c|c|c|c|c|c|}
\hline Compound & $R^{\prime}$ & $R^{\prime \prime}$ & $c \log P^{\mathrm{a}}$ & $D A T K_{i}(n M)$ & DA uptake inhibition $I C_{50}(n M)$ \\
\hline $3-\mathrm{Cl} \mathrm{BZT}$ & $3^{\prime}-\mathrm{Cl}$ & $\mathrm{H}$ & 4.35 & $21.6 \pm 1.51$ & $12.5 \pm 0.91$ \\
\hline 4-Cl BZT & $4-\mathrm{Cl}$ & $\mathrm{H}$ & 4.35 & $30.0 \pm 3.60$ & $23.1 \pm 1.80$ \\
\hline 3’,4’-di Cl BZT & $3^{\prime}-\mathrm{Cl}$ & $4{ }^{\prime}-\mathrm{Cl}$ & 5.07 & $32.5 \pm 4.88$ & $12.3 \pm 1.13$ \\
\hline 4,4“-di Cl BZT & $4^{\prime}-\mathrm{Cl}$ & 4 - $-\mathrm{Cl}$ & 5.07 & $20.0 \pm 2.80$ & $23.4 \pm 3.00$ \\
\hline
\end{tabular}

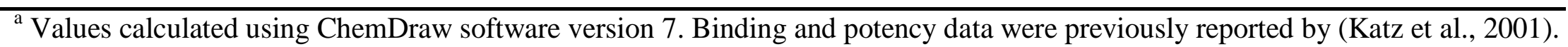


Table 2. Permeability values and efflux ratios of the chloro BZT analogs across MDCKMDR1 and Caco-2 monolayers at a concentration of $0.1 \mathrm{mM}$.

\begin{tabular}{|c|c|c|c|c|c|c|}
\hline \multirow[t]{3}{*}{ Compound } & \multicolumn{3}{|c|}{ MDCK-MDR1 } & \multicolumn{3}{|c|}{ Caco-2 } \\
\hline & \multicolumn{2}{|c|}{$\mathrm{P}_{\text {app }}\left({\left.\mathrm{x} 10^{-6} \mathrm{~cm} / \mathrm{sec}\right)}\right.$} & \multirow{2}{*}{$\begin{array}{l}\text { Efflux } \\
\text { Ratio }\end{array}$} & \multicolumn{2}{|c|}{$\mathrm{P}_{\text {app }}\left(\mathrm{x} 10^{-6} \mathrm{~cm} / \mathrm{sec}\right)$} & \multirow{2}{*}{$\begin{array}{l}\text { Efflux } \\
\text { Ratio }\end{array}$} \\
\hline & A-B & B-A & & A-B & B-A & \\
\hline 3'-Cl BZT & $32.23 \pm 2.28$ & $68.04 \pm 2.75$ & 2.1 & $21.65 \pm 0.89$ & $71.92 \pm 0.53$ & 3.3 \\
\hline 4-Cl BZT & $30.98 \pm 0.64$ & $66.41 \pm 7.18$ & 2.1 & $12.22 \pm 1.53$ & $45.39 \pm 1.71$ & 3.7 \\
\hline 3,4“'-di Cl BZT & $16.09 \pm 1.10$ & $49.17 \pm 4.89$ & 3.1 & $2.54 \pm 0.33$ & $9.98 \pm 1.53$ & 3.9 \\
\hline 4,4“’-di Cl BZT & $8.26 \pm 0.68$ & $57.17 \pm 7.55$ & 6.9 & $1.37 \pm 0.18$ & $38.93 \pm 2.42$ & 28.4 \\
\hline
\end{tabular}


Table 3. Intrinsic clearance values for 4`,4“- $\operatorname{diCl} \mathrm{BZT}(3 \mu \mathrm{M})$ in human and male Sprague Dawley rat pooled liver microsomes $(0.8 \mathrm{mg} / \mathrm{ml})$ and in human and rat recombinant CYPs (50 pmol/ml).

\begin{tabular}{lc}
\hline Microsomal Preparation & Intrinsic Clearance $^{1}$ \\
\hline Pooled HLM & $12.75 \pm 2.65$ \\
Pooled male RLM & $21.46 \pm 1.39$ \\
Human CYP 2C19 & $0.07 \pm 0.01^{b}$ \\
Human CYP 2D6 & $0.04 \pm 0.01^{b}$ \\
Rat CYP 2C11 & $1.46 \pm 0.39^{a}$ \\
Rat CYP 3A1 & $0.08 \pm 0.01^{b}$ \\
\hline
\end{tabular}

${ }^{1}$ Intrinsic clearance is reported as $\mu \mathrm{l} / \mathrm{min} / \mathrm{mg}$ protein for HLM and RLM and as $\mu 1 / \mathrm{min} / \mathrm{pmol}$ CYP for the recombinant CYPs ( $\mathrm{n}=3 /$ reaction). The intrinsic clearance values in the recombinant CYPs that share the same letter are not significantly different at $\alpha=0.05$. 
Table 4. NONMEM estimated population pharmacokinetic parameters for 4`-Cl BZT $(\mathrm{n}=42)$ and 4`,4“-diCl BZT (n= 54) along with their associated inter-animal variability (IAV) upon i.v. administration to Sprague-Dawley rats.

\begin{tabular}{|c|c|c|c|c|}
\hline \multirow[b]{2}{*}{ Parameter } & \multicolumn{2}{|c|}{ 4-CI BZT } & \multicolumn{2}{|c|}{$4,4 “-d i C l$ BZT } \\
\hline & Estimate (CV \%) & IAV \% (CV \%) & Estimate (CV \%) & IAV \% (CV \%) \\
\hline CL (L/hr/kg) & $4.7(5.8)$ & $-{ }^{a}$ & $0.6(14.9)$ & $53.1(42.9)$ \\
\hline $\mathrm{Vc}(\mathrm{L})$ & 4.7 (14.3) & $21.5(136.7)$ & $5.3(11.2)$ & $21.2(84.2)$ \\
\hline $\mathrm{Vp}(\mathrm{L})$ & $16.9(14.1)$ & $45.6(69.2)$ & $12.8(6.5)$ & $--^{a}$ \\
\hline $\mathrm{Q}(\mathrm{L} / \mathrm{hr} / \mathrm{kg})$ & $13.0(23.3)$ & $54.7(62.2)$ & $10.9(12.8)$ & $37.4(63.4)$ \\
\hline
\end{tabular}

CL: clearance. Vc: volume of the central compartment. Vp: volume of the peripheral compartment. Q: inter-compartmental clearance. ${ }^{a}$ Not significantly different from zero $(\mathrm{p}>0.05)$ based on the Likelihood Ratio Test (The objective function was compared when the parameter was freely estimated and when it was fixed to zero) . 
Table 5. Secondary pharmacokinetic parameters (calculated from the NONMEM empirical Bayes estimates of the individual animalspecific PK parameters) and brain to plasma partition coefficient and brain $\mathrm{t}_{1 / 2}$ (based on the non-compartmental analysis performed with WinNonlin ) for 4`- $\mathrm{Cl}$ BZT and 4`4“`-diCl BZT in comparison to previously published data from our laboratory for cocaine (Raje et al., 2003).

\begin{tabular}{|c|c|c|c|c|c|}
\hline & \multicolumn{2}{|c|}{ 4-CI BZT } & \multicolumn{2}{|c|}{$4,4 ’-d i C l$ BZT } & \multirow{2}{*}{$\begin{array}{c}\text { Cocaine } \\
5 \mathrm{mg} / \mathrm{kg}\end{array}$} \\
\hline & $5 \mathrm{mg} / \mathrm{kg}$ & $10 \mathrm{mg} / \mathrm{kg}$ & $5 \mathrm{mg} / \mathrm{kg}$ & $10 \mathrm{mg} / \mathrm{kg}$ & \\
\hline Parameter & $\mathrm{n}=18$ & $\mathrm{n}=24$ & $\mathrm{n}=27$ & $\mathrm{n}=27$ & $\mathrm{n}=24$ \\
\hline$\overline{\mathrm{AUC}}$ & 63.3 & 126.6 & $506.6 \pm 153.5$ & $1229.6 \pm 362.5$ & $98.9 \pm 7.4$ \\
\hline \multicolumn{6}{|l|}{$(\mu \mathrm{g} * \min / \mathrm{ml})$} \\
\hline Vss (L) & $24.1 \pm 3.6^{a}$ & $21.6 \pm 4.4$ & $18.1 \pm 0.2^{a, b}$ & $17.9 \pm 0.5^{b}$ & $0.9 \pm 0.1$ \\
\hline $\mathrm{t}_{1 / 2}(\mathrm{hr})$ & $3.52 \pm 0.5^{a}$ & $3.16 \pm 0.7$ & $21.1 \pm 6.2^{a, b}$ & $25.3 \pm 7.0^{b}$ & $0.49 \pm 0.06$ \\
\hline$\overline{\mathrm{Ri}}$ & 4.6 & 3.1 & 4.5 & 4.7 & 2.1 \\
\hline Brain $\mathrm{t}_{1 / 2}(\mathrm{hr})$ & 3.9 & 5.1 & 9.7 & 12.5 & 0.6 \\
\hline
\end{tabular}

${ }^{a}$ Statistically significant difference $(\mathrm{p}<0.05)$ from the corresponding parameter of the equivalent dose of cocaine.

${ }^{b}$ Statistically significant difference $(\mathrm{p}<0.001)$ from the corresponding parameter of the equivalent dose of $4{ }^{`}-\mathrm{Cl} \mathrm{BZT}$. 
$\mathrm{H}_{3} \mathrm{C}_{1}$

$\mathrm{N}$
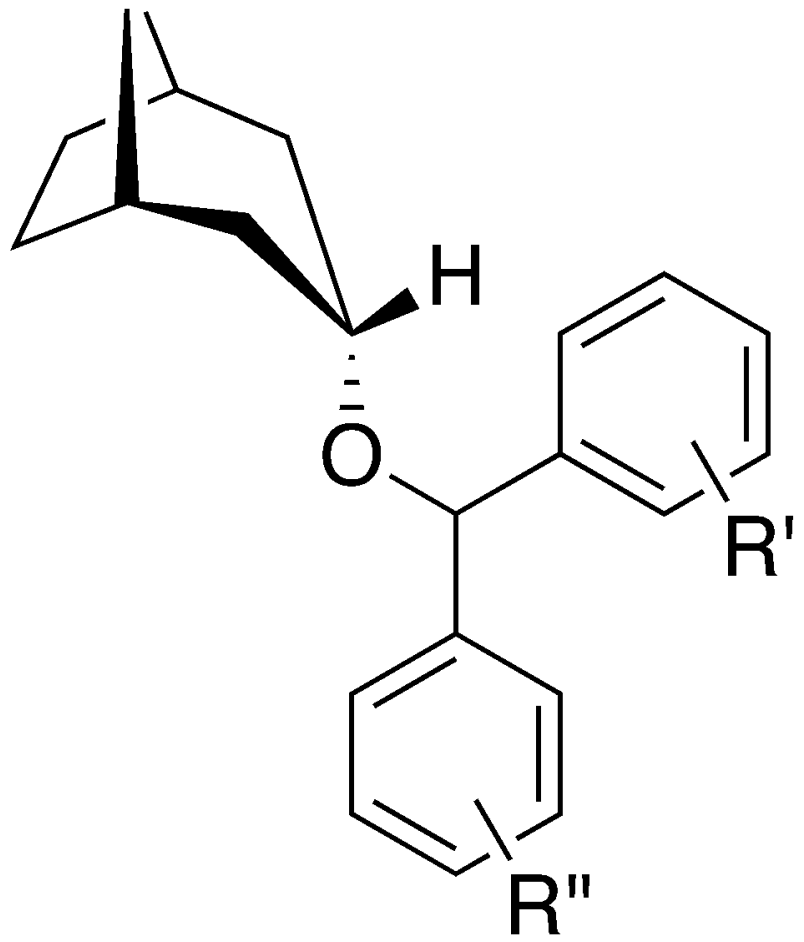

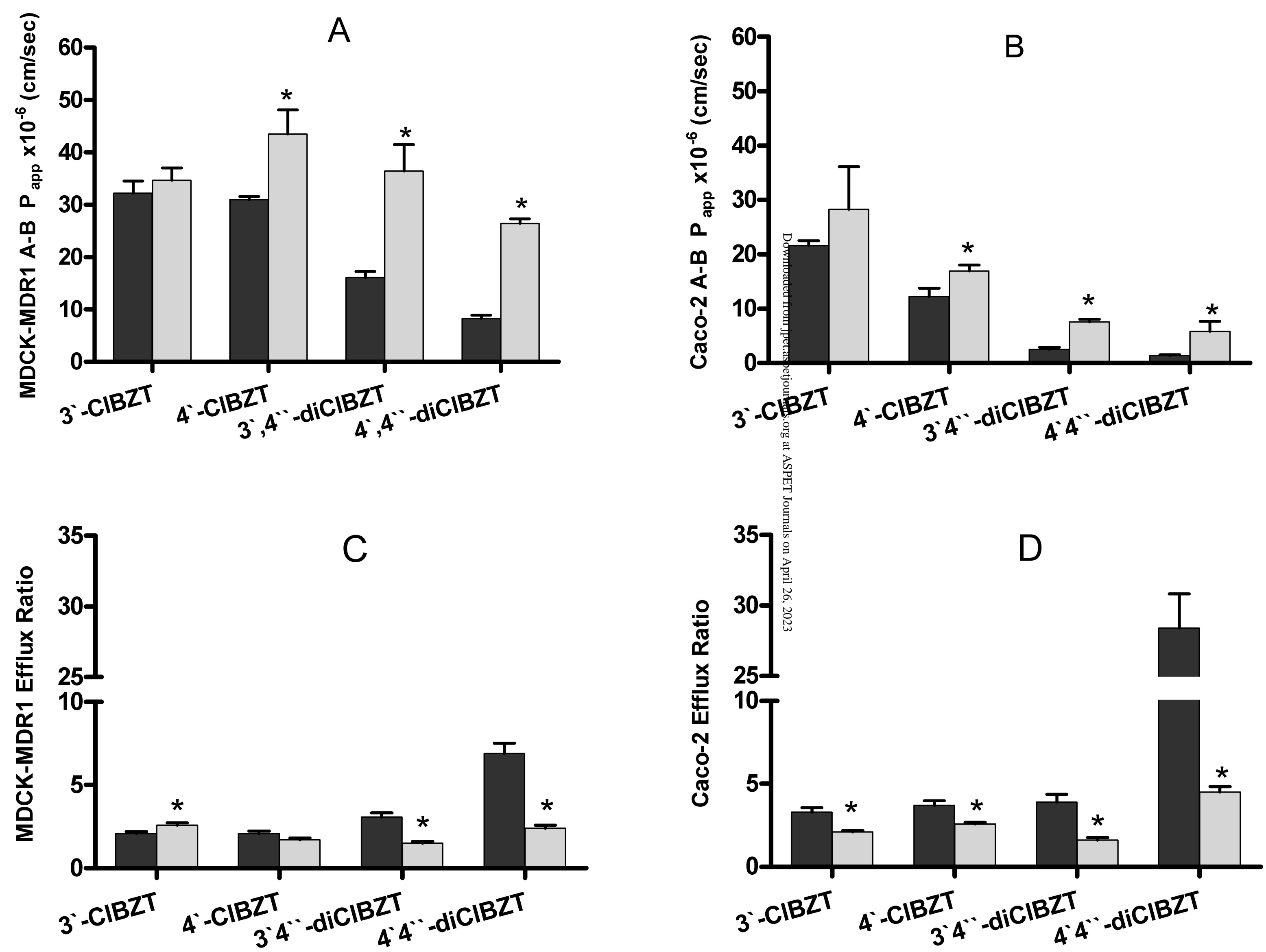

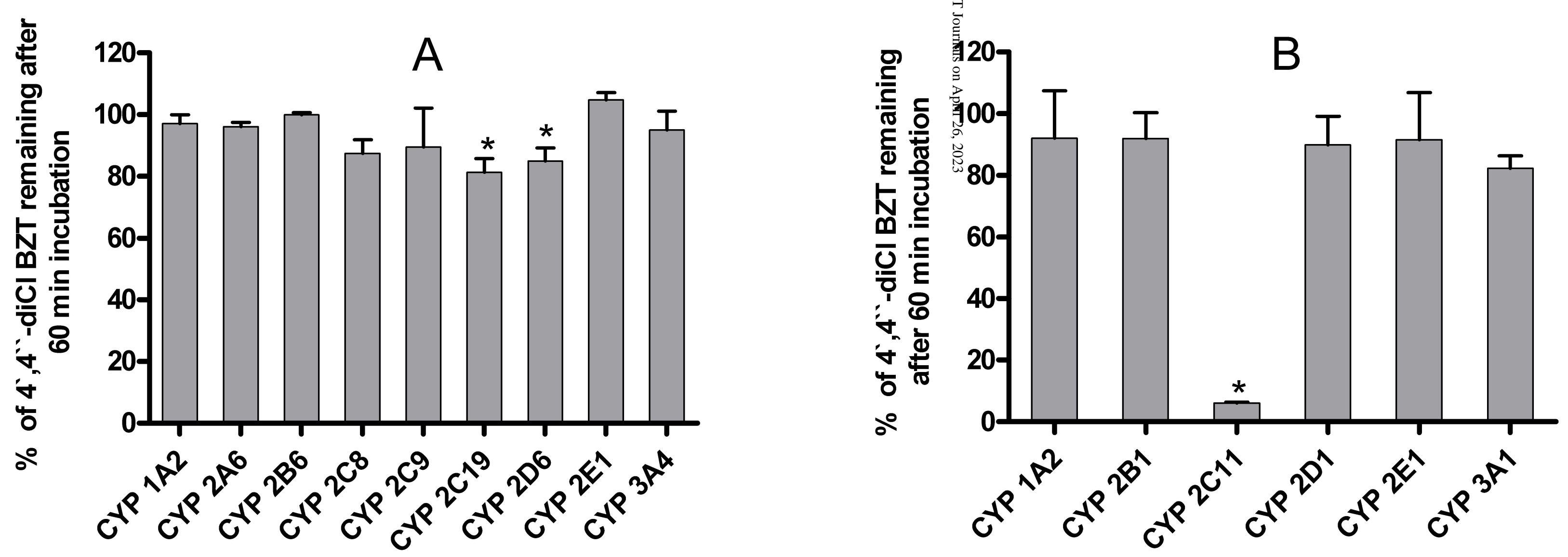


\section{A}

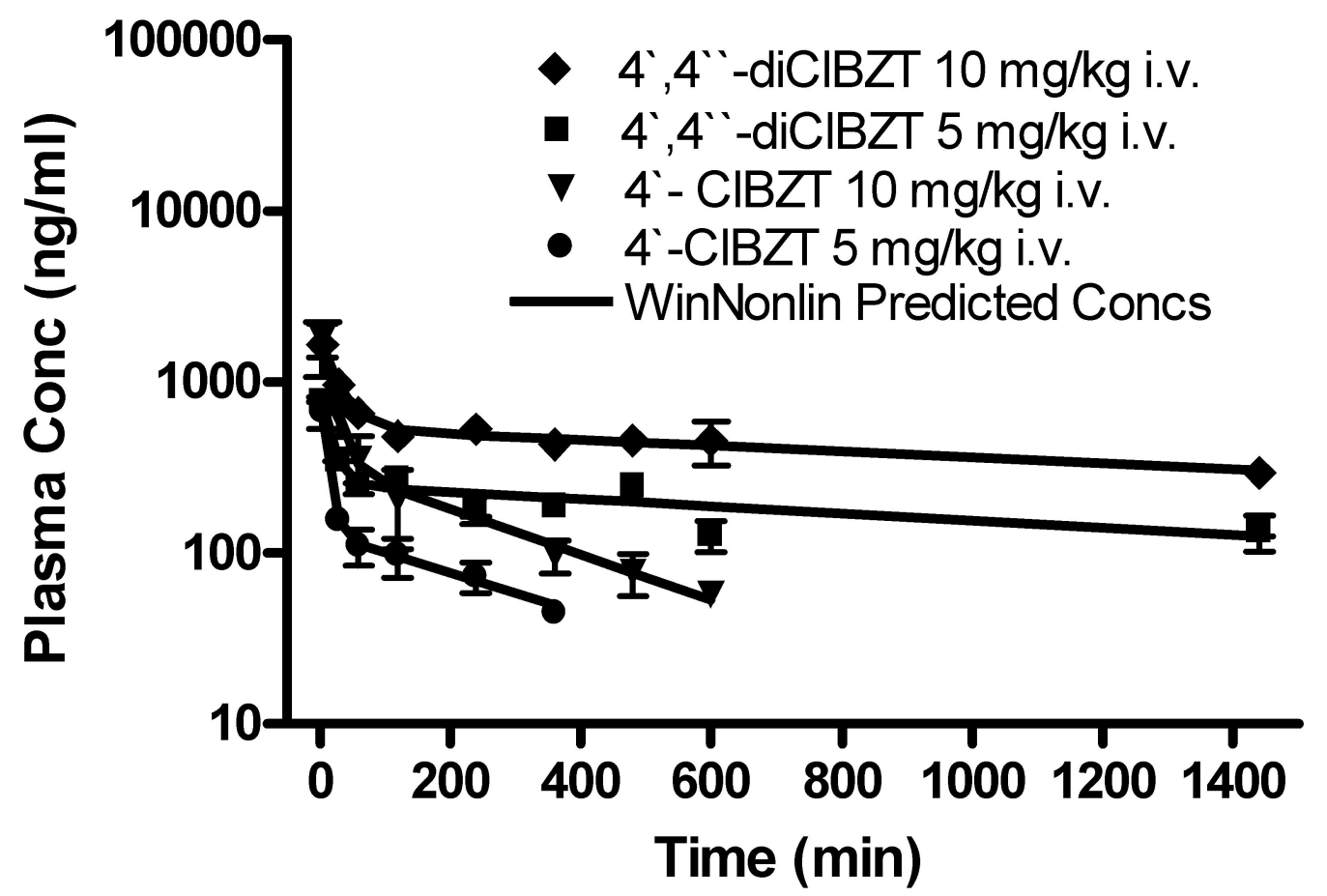

B

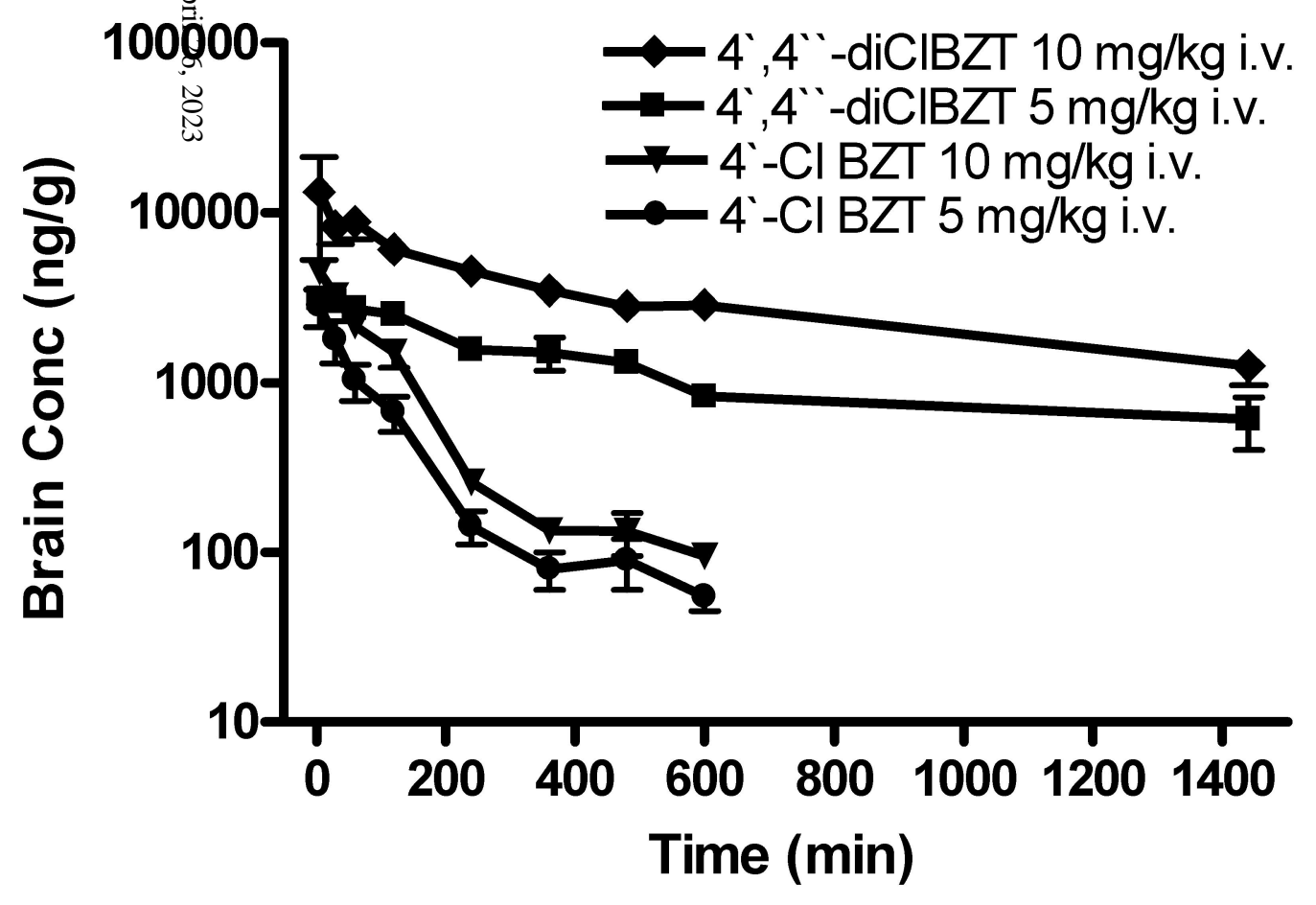




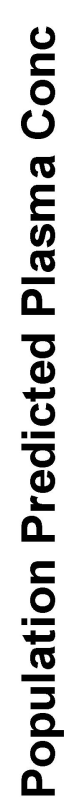

है

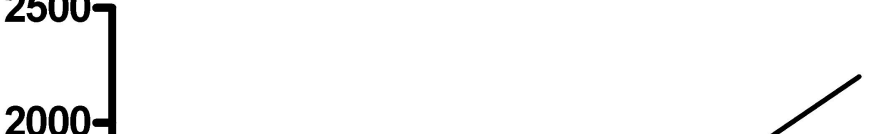

A

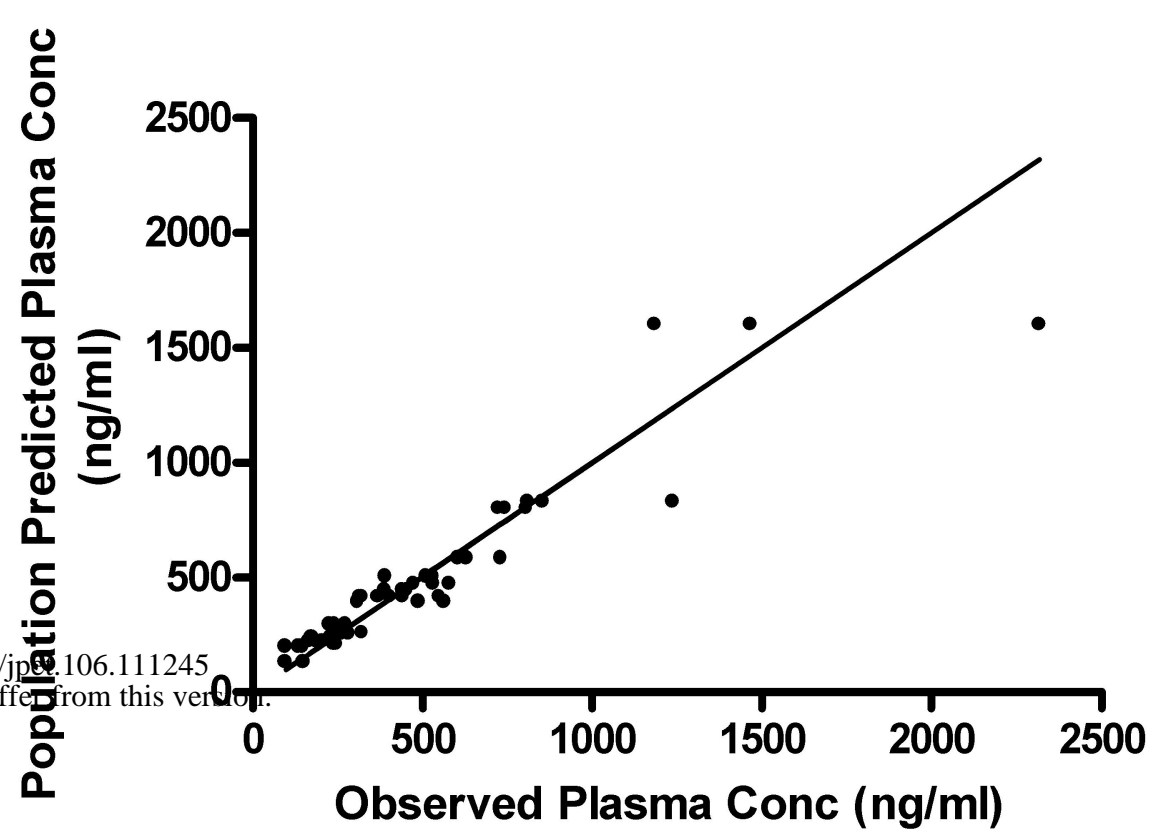

B

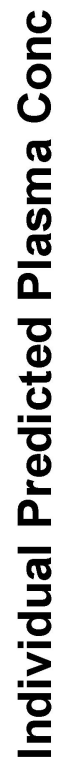
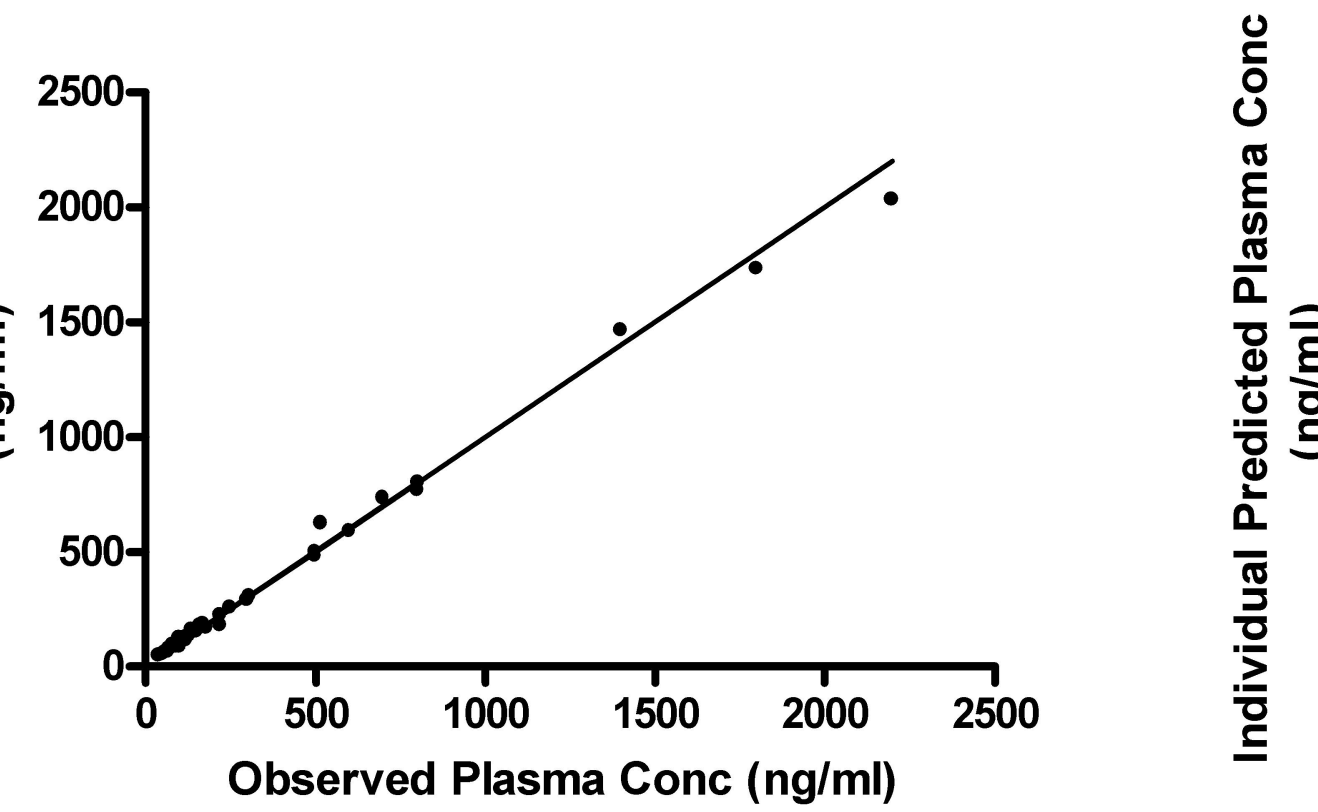

C
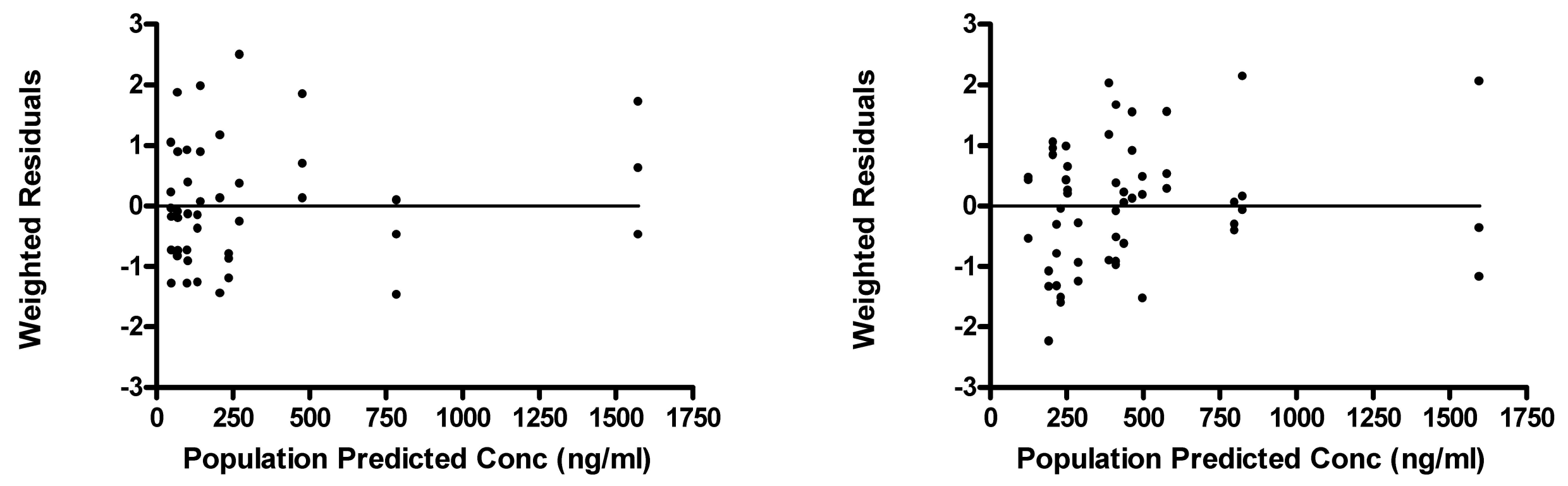